\title{
Do we use visual codes when information is not presented visually?
}

\section{Dominic Guitard ${ }^{1} \cdot$ Nelson Cowan ${ }^{2}$}

Published online: 3 June 2020

(C) The Psychonomic Society, Inc. 2020

\begin{abstract}
For many years, the working/short-term memory literature has been dominated by the study of phonological codes. Consequently, insufficient attention has been devoted to visual codes. In the present study, we attempt to remedy the situation by exploring a critical aspect of modern models of working memory, namely the principle that responses do not depend primarily on what kinds of materials are presented, but on what kinds of codes are generated from those materials. More specifically, we used the visual similarity effect as a tool to ask whether there is a generation of visual codes when information is not presented visually. In two immediate serial recall experiments, we manipulated the visual similarity (similar words, dissimilar words), the presentation modality (visual presentation, auditory presentation), and concurrent articulation (none, concurrent articulation). We observed a visual similarity effect independent of presentation modality. Comparable results were observed with two different sets of stimuli and with or without concurrent articulation. Thus, for the first time, we demonstrate that, from acoustically presented word lists, visual codes in working/short-term memory are generated, producing a visual similarity effect. It is now clear that the encoding of visual or acoustic presentation to include the opposite type of representation is bidirectional.
\end{abstract}

Keywords Visual similarity $\cdot$ Short-term memory $\cdot$ Presentation modality $\cdot$ Immediate serial recall

\section{Introduction}

When participants must recall a sequence of items just after their presentation, performance is superior if the to-beremembered items are dissimilar from one another. Manipulating the similarity of to-be-remembered items has been a critical tool for the development of memory models (Crowder, 1979). The rich history of similarity manipulation in the memory literature has provided a valuable insight into the underlying codes of a memory representation. To better understand why manipulation of similarity of to-beremembered items is so valuable, one can imagine the following experiments. A researcher presents two series of items to a participant (e.g., words, pictures, or sounds), one in which all items of the lists share some features (e.g., letters or sounds)

Dominic Guitard edg2851@umoncton.ca

$\bowtie$ Nelson Cowan cowann@missouri.edu

1 Faculte des Sciences de la Sante et des Services Communautaires, Department of Psychology, Université de Moncton, Moncton, New Brunswick, Canada

2 Department of Psychological Sciences, University of Missouri, Columbia, MO, USA and one in which no items in the lists share such features. When comparing memory performance, if the performance on lists sharing some features is inferior to the performance on lists not sharing features, it suggests that these features must somehow be used to represent information in memory. Importantly, the features need not be intrinsic to the stimuli, but can be features added by the perceiver. Thus, in the memory literature, most researchers of similarity effects have focused on phonological codes for verbal items even though many presented information visually, in printed form (starting with Conrad, 1964). In the present study, we do the converse, exploring whether visual codes are used when information is not presented visually.

One of the most extensively studied effects of similarity in memory literature is the phonological similarity effect, also known as the acoustic similarity effect (Baddeley, 1966; V. Coltheart, 1993; Conrad, 1964; Conrad \& Hull, 1964; Crowder, 1979; Farrell \& Lewandowsky, 2003; Poirier \& Saint-Aubin, 1996; Wickelgren, 1965). This effect refers to the superior memory performance for dissimilar sounding items (e.g., duck, lace, moon, pies, road) compared to similar sounding items (e.g., rang, tang, gang, sang, pang). This effect is a cornerstone of a seminal working memory model (Baddeley \& Hitch, 1974) and has played a major role in the development of computational models of working memory 
(e.g., Burgess \& Hitch, 1999; Henson, 1998; Lewandowsky \& Farrell, 2008). The acoustic similarity effect is now widely accepted as the benchmark memory effect in short-term memory (Oberauer et al., 2018).

The acoustic similarity effect interacts with concurrent articulation and the modality of presentation (Peterson \& Johnson, 1971). More specifically, the acoustic similarity is eliminated by concurrent articulation of a word irrelevant to the task if the information is presented visually, though it remains in a diminished form if the information is presented acoustically (Baddeley, Lewis, \& Vallar, 1984; Cowan, Cartwright, Winterowd, \& Sherk, 1987; Peterson \& Johnson, 1971). These types of findings support key aspects of the working memory model (Baddeley et al., 1984), in particular phonological coding that occurs automatically for acoustic presentation but only with dedicated articulation for visual presentation.

Despite the numerous beneficial implications of studying the acoustic similarity effect, it led researchers to disproportionally focus on phonological codes. Consequently, far less attention has been devoted to other codes, such as visual codes, as highlighted by Hurlstone, Hitch, and Baddeley (2014) in their overview of memory for serial order. An exception is recent work on the visual similarity effect. This effect refers to the better recall of visually dissimilar items (Logie, Della Sala, Wynn, \& Baddeley, 2000). The visual similarity effect is also considered by researchers as a benchmark effect (Oberauer et al., 2018) despite being far less studied than phonological similarity. The effects of visual similarity have gained some interest in recent years and have been observed with a variety of manipulations, not only of orthography (Chubala, Guitard, Neath, Saint-Aubin, \& Surprenant, 2020; Logie et al., 2000; Logie, Saito, Morita, Varma, \& Norris, 2016; Saito, Logie, Morita, \& Law, 2008), but also of visual patterns and faces (Avons \& Mason, 1999; Poirier, Saint-Aubin, Musselwhite, Mohanadas, \& Mahammed, 2007; Smyth, Hay, Hitch, \& Horton, 2005). The visual similarity effect for words has played an important role in the development of memory models such as the Primacy Model (see, e.g., Logie et al., 2016) and the Feature Model (see, e.g., Chubala et al., 2020).

Here, in two experiments, we focus on better understanding visual codes. More specifically, we focus on an important aspect of modern models of working memory: that they posit that responses do not depend primarily on what kinds of materials were presented, but on what kinds of codes are generated from those materials. The generation of phonological codes for printed materials has been amply tested, as we have noted. However, the generation of visual, orthographic codes for acoustic materials has not been properly discussed or tested until now.

The current work follows the footsteps of pioneer work on dual coding by Paivio $(1971,1991,2007)$, in which verbal and visual codes can be generated from both verbal and nonverbal materials. Unlike the dual-coding theory in which visual codes can refer to semantic visual images, though, the present study focuses on visual orthographic codes. Both lines of research are driven by a similar assumption that the features need not be intrinsic to the stimuli, but can be added by the perceiver.

This study has major implications that will help to refine theories of memory. Without proper investigation of the generation of visual codes from acoustic materials, one could envision versions of each theory that are asymmetrical, in that conversion would go only from a secondary modality for language (vision, orthography) to a primary modality (acoustics, phonology), as discussed in the primary linguistic hypothesis of Shand and Klima (1981), and not in the reverse direction. In contrast, from the point of view of general models of cognition, one can see why there could be symmetry in the conversion of codes. A general principle of cognition is that the response to a stimulus depends not on the nature of the stimulus per se but on how it is interpreted relative to what is known. As an example, a sound following the last sound in a spoken list impairs memory for the list more if it is perceived as speech too compared to when the same sound is perceived as non-human (e.g., a human sound [baa] vs. a sheep bleating [baa]) (Ayres, Jonides, Reitman, Egan, \& Howard, 1979; Neath, Surprenant, \& Crowder, 1993). The internal codes that are generated (speech vs. nonspeech) determine how the item is processed. The present application of the internal codes principle would be that, in addition to converting printed words into an acoustic code corresponding to that word, for example, "sounding out" the word, people may generate the visual image corresponding to the printed version of a spoken word, for example, mentally print the word. Moreover, people may use both codes to facilitate immediate recall. Thus, there are theoretical vantagepoints from which one could expect the absence or presence of visual similarity effects for the immediate recall of spoken word lists.

There have been recent studies that have invoked models of serial recall to account for visual similarity effects with visual materials (the Feature Model of Nairne, 1990: Chubala et al., 2020; the Primacy Model of Page \& Norris, 1998: Logie et al., 2016). These works have not extended the theories to predict the absence or presence of visual similarity effects for acoustic materials but, we believe, such data would at least help to constrain the theories. (1) In the feature model, there is no fundamental difference between one modality and another except for their modality-specific features. There are amodal features of phonology that can be generated from either spoken or printed words and, similarly, there are amodal features of orthography that can be generated from either spoken or printed words. In either case, amodal features of one word can overwrite similar features within another word, so that the visual similarity between the printed counterparts of spoken 
words could be detrimental to recall. On the other hand, the theorists have not specifically stated that amodal orthographic features are used in serial recall of words. (2) In the primacy model, words have a degree of activation with a primacy gradient that feeds into a second, output phase of recall. In that output phase, similarity of phonological features can lead to confusion (Page \& Norris, 1998) and, according to a recent extension of the model, so can the similarity of visual features (Logie et al., 2016). Yet, from this point of view no one has stated whether visual features are formed from acoustic input.

In the present study, we used the visual similarity effect to investigate visual codes in memory for words using both visual and auditory presentation. Despite the recent growing interest in the visual similarity effect, as previously mentioned, the generation of visual codes for acoustic materials has not been adequately investigated. To our knowledge, only Baddeley (Experiment II, 1966) has explored the visual similarity effect in serial recall with an auditory presentation. Baddeley compared performance on three sets of five words (sets similar acoustically, similar visually, and dissimilar both acoustically and visually). At the descriptive level, memory performance was inferior for the visually similar set $(M=$ $55.8 \%)$ compared to the acoustically and visually dissimilar set $(M=63.5 \%)$. However, this contrast did not reach significance. One potential factor might be that the study was underpowered $(\mathrm{N}=17)$. Even if an effect had been obtained with these words, the basis of the effect would be unclear. Their visually similar list items (rough, cough, through, dough, bough) were arguably more phonologically similar than the control list (plea, friend, sleigh, row, board), making the comparison less than ideal. In this study, as can be seen below, we took great care to control for an extensive number of factors, and we increased the sample size to ensure sufficient power of our manipulation (see Method sections for more details).

In summary, in the present study, we examine if visual codes support the memory representation of both visual and auditory word lists in immediate-recall experiments. The logic for this study is analogous to the logic by which Conrad (1964) first examined acoustic similarity effects for printed materials. Specifically, this cross-modal kind of procedure can assess whether a stimulus presented in one modality gives rise to a mental code based on another modality. Just as Conrad found that printed words give rise to an internal acoustic code, spoken words could give rise to an internal orthographic code. In two experiments with different sets of stimuli, we manipulated the visual similarity of English words' orthography, using either visual or acoustic presentation.

The rationale for replicating our findings with two sets of stimuli is that the memory literature has been seriously affected in the past by the use of small pools of stimuli, which increases the likelihood that idiosyncratic characteristics of stimuli cause an atypical pattern of results (see, e.g., Caplan, Rochon, \& Waters, 1992; Guitard, Miller, Neath, \&
Roodenrys, 2019; Lovatt, Avons, \& Masterson, 2000; Neath, Bireta, \& Surprenant, 2003). Using two sets of stimuli in different experiments increases the strength of our findings.

We used concurrent articulation on some trials in both experiments. The purpose of using it was to determine boundary conditions of when visual similarity has an effect, for further refinement in theories. According to one possible theoretical view, the phonological information would take precedence when it is available, and the visual information would only be used as a backup when phonological information is not sufficient. With such a view, the effect of visual similarity should be larger with concurrent articulation than without it at least in the visual modality, inasmuch as concurrent articulation should prevent formation of the phonological code. A similar purpose for concurrent articulation has been reported previously (Hitch, Woodin, \& Baker, 1989; Poirier et al., 2007). In contrast, if phonological and visual codes are used in parallel within a trial, picking up on complementary types of information that can boost recall independently, then the effect of visual similarity should not depend on the presence or absence of concurrent articulation.

Although many models do not specify which of these two relations between visual and phonological codes is the case, the independent use of codes seems more consistent than a dependent, contingent use of the visual code in the spirit of models focusing on many concurrent, activated features, such as the Feature Model (Chubala et al., 2020; Nairne, 1990) and the embedded processes model (Cowan, 1988, 2019). Later we will pursue other possible implications of our results for models of serial order recall.

\section{Experiment 1}

Experiment 1 was designed to examine if visual codes support the memory representation of both visual and auditory word lists in immediate recall. To do so we used visual similarity as a tool analogous to the logic by which Conrad (1964) first examined acoustic similarity effects for printed materials. More specifically, in this experiment participants memorized sequence of visually similar or dissimilar words. The words were presented visually for half of the trials, versus through headphones for the other half. Concurrent articulation was required during encoding on half of the trials. The purpose of using it, as previously mentioned, was to determine boundary conditions of when visual similarity has an effect, for a further refinement in theories.

\section{Method}

Participants Forty undergraduate students volunteered from University of Missouri to participate for course credits. The mean age of the participants was 18.67 years $(\mathrm{SD}=1.20$, 
range 18-25); 23 self-identified as a female and 17 as a male. All participants were native speakers of English.

For both experiments we used G*Power (Faul, Erdfelder, Buchner, \& Lang, 2009) to determine our sample size. We determined that a sample size of 40 would have power of 0.87 to detect a medium effect size $(d=.50)$. The selection of a medium effect size is conservative as in the literature the effect size of the visual similarity effect has been slightly larger. For instance, using the same stimuli, Chubala et al. (Experiment III, 2020) found an effect size of 1.10. The conservative selection in the present study was done to maximize the possibility of detecting a visual similarity effect with both modalities.

Materials All experiments were programmed with E-Prime 2.0. All words and texts, unless otherwise mentioned, were presented in black and bold, uppercase, $24 \mathrm{pt}$. Courier font, at the center of a computer screen on a white background.

The stimuli were taken from Experiment 3 of Chubala et al. (2020). The visually dissimilar words were CROW, CURL, CZAR, SHIP, TALE, and UNDO. The visually similar words were CORE, CUBE, CURB, DOPE, OGRE, and PURE. The stimuli were created by Chubala et al. using the visual similarity matrix for Latin-based alphabets of Simpson, Mousikou, Montoya, and Defior (2013). The stimuli were also equated on phonological similarity using a measure based on PSIMETRICA, a measure of phonological dissimilarity (Mueller, Seymour, Kieras, \& Meyer, 2003). Using standard American pronunciation, the mean phonological dissimilarity scores were equivalent between the visually similar lists and the visually dissimilar lists. The stimuli were also controlled for several other dimensions (see Chubala et al. for more details). The selection of the stimuli of the Experiment 3 of Chubala and colleagues was motivated by numerous factors. For instance, the stimuli were highly controlled for a variety of psycholinguistic factors such as word frequency, neighborhood size, phonological similarity, and many more, the stimuli set yield a large visual similarity effect and the stimuli were in English, the native language of our participants. Given all these factors, we believe that their stimuli were optimal for a first exploration of the visual similarity effect with a non-visual presentation.

The audio files for both experiments were created with the free version of Google Cloud Text-to-Speech (https://cloud. google.com/text-to-speech/) and the sound files were recorded and fitted in a standard-duration window of $1,000 \mathrm{~ms}$ with Audacity 2.3.0 (https://www.audacityteam.org/). The following options were selected to create the sound files with Google Cloud Text-to-Speech: language $=$ English $($ USA $)$; voice type $=$ WaveNet; voice name $=$ en-USWavenet-D; audio device profile $=$ headphone or earbuds; speed $=1 ;$ pitch $=0$. All audio files were in a male voice.
Design A $2 \times 2 \times 2 \times 6$ repeated-measure design was implemented with the following factors: visual similarity (similar, dissimilar), presentation modality (visual, auditory), articulation (none, concurrent articulation) and serial position (1-6). The experiment was divided into two blocks of four practice trials and 32 experimental trials, each corresponding to a presentation modality (visual, auditory). The order of the two blocks was counterbalanced across participants. Therefore, for both experiments, half of the participants started with a visual presentation and half of the participants started with an auditory presentation. In each block, there were eight experimental trials for each of the four possible conditions (i.e., visually similar without concurrent articulation, visually similar with concurrent articulation, visually dissimilar without concurrent articulation, visually dissimilar with concurrent articulation) preceded by one practice trial of each of the four conditions. The six available words were presented in a random order on each trial. The order of the conditions (visually similar without concurrent articulation, visually similar with concurrent articulation, visually dissimilar without concurrent articulation, visually dissimilar with concurrent articulation) was randomized within each block (visual presentation, auditory presentation) and each participant.

Procedure All participants were tested in one experimental session lasting approximately $45 \mathrm{~min}$ in a sound-attenuated booth. Experimenters monitored participants to ensure compliance with the instruction. Each session started with a familiarization of the words and of the articulation task. For the familiarization of words, there were four trials each starting with a fixation crossed for $1,000 \mathrm{~ms}$, immediately followed by presentation of the six words presented at a rate of one word per 1,000 ms (1,000 ms on, $0 \mathrm{~ms}$ off). The words were presented visually at the center of the screen and through headphones. There were two trials for the visually similar lists and two trials for the visually dissimilar lists. During the familiarization of the word, the participants were asked to look at each word as it appears. For the familiarization of the concurrent articulation, participants had to whisper the word "THE" at the rate of two words per second 24 times under the supervision of the experimenter. The rate of two words per second for concurrent articulation was demonstrated by the presentation of the word "THE" on the center of the screen at the rate of two words per second. Following the familiarization, participants started the main experiment.

Each trial began with the presentation of the instruction for the articulation condition, which was presented until the participant pressed the "space bar" key of the keyboard. The instruction was either "silence" for the trials without the concurrent articulation or "speaking" for the trials with the concurrent articulation. When concurrent articulation was required, participants had to whisper the word "THE" at the rate of two words per second during the presentation of the words. 
Immediately after, a fixation crossed was presented for $1,000 \mathrm{~ms}$ followed by the presentation of the six to-beremembered words presented at a rate of one word per $1,000 \mathrm{~ms}$ (1,000 ms on, $0 \mathrm{~ms}$ off). Recall was indicated $1,000 \mathrm{~ms}$ after the presentation of the last word in the upper part of the screen by three questions marks in red. Participants were instructed to recall the words from the first to the last word that has been presented. Following the procedure of Chubala et al. (2020), recall was achieved by keying each word on the keyboard. After each word participants had to press the "space bar" to register their response. No time limit was imposed for recall, and participants were not allowed to backtrack in order to change a previous response. Participants were instructed to key the word "SKIP" when they were unable to recall a given word. Participants initiated the next trial by pressing the mouse. The procedure was as described for both visual and auditory presentation with the exception that, for visual blocks, words were only presented at the center of the screen, and for auditory blocks, words were only presented through headphones.

\section{Data analysis}

A strict serial recall criterion was used. With this criterion, words must be recalled in their presentation position to be considered correct. The proportion of correct responses was assessed as a function of visual similarity, presentation modality, articulation, and serial position. Effects involving position are not of theoretical interest to the present study and were included in the analyses only to account for variance that would otherwise go into the error term. Therefore, effects involving position are mentioned in the tables but not in the text. For all analyses, raw data were used, meaning when the typed responses of the participants were not an exact match to the presented word, the word was counted as wrong. ${ }^{1}$

\footnotetext{
${ }^{1}$ In a different analysis method, typed responses were checked for errors such as omitting an initial letter (URL rather than CURL), omitting a final letter (CUB rather than CUBE), transpositions (CRUB rather than CURB), and so on. A total of 331 out of 5,922 responses (5. 59\%) were changed, 202 in the dissimilar condition and 129 in the similar condition. We decided to use uncorrected data for analyses reported in both experiments as it was difficult to distinguish between typing errors and genuine incorrect attempts to recall an item. For instance, a participant could type CUB and it could refer to CUBE or CURB. To remain transparent, one notable change between uncorrected and corrected data in the first experiment is the presence of marginal two-way interaction between similarity and articulation with corrected data, $F(1,39)=$ $4.73, \eta_{p}^{2}=.11, p=.036, \mathrm{BF}_{10}=8.27$, which failed to reach a conventional level of significance with uncorrected data, $F(1,39)=2.97, \eta_{p}^{2}=.07, p=$ $.093, \mathrm{BF}_{10}=0.88$. This interaction, as can be seen in Fig. 1, reflects a floor effect observed for the end of the list items under concurrent articulation (particularly apparent in the visual presentation condition). This interaction between similarity and articulation has also been documented in the past by Poirier et al. (Experiment 1, 2007). Like the current study, the interaction was attributed to the overall performance level (ceiling effect in their case). Taken together, and considering the results of Experiment 2, we believe that the absence of the two-way interaction is more credible than the presence.
}

To analyze our data according to the potentially different preferences of readers, we used both frequentist and Bayesian techniques using R (Version 3.6.1; R Core Team, 2019) and JASP (JASP Team, 2019 version 0.11.1.0). For the frequentist approach, we report $p$ values and effect size. The .05 level of significance was adopted and the Greenhouse-Geisser correction was applied when the sphericity criterion was violated. For the Bayesian approach, we report Bayes factors (BFs), the evidence ratio between two competing models, which provides evidence in support of the denominator model (subscripted as 01 ) or the alternative model in the numerator (subscripted as 10). The BF interpretation is guided by the benchmarks taken from Kass and Raftery (1995): BF $<3$ indicates weak or anecdotal evidence; $3 \leq \mathrm{BF}<20$ indicates positive evidence; $20 \leq \mathrm{BF}<150$ indicates strong evidence; $\mathrm{BF}>150$ indicates very strong evidence. For all Bayesian analysis, we used BayesFactor package and the default prior (Version 0.9.12-4.2; see Morey \& Rouder, 2018; Rouder, Morey, Speckman, \& Province, 2012; Rouder, Speckman, Sun, Morey, \& Iverson, 2009). Main-effect models were compared to the intercept-only model (Subject) and interaction models were compared to the main-effects model (Similarity + Modality + Articulation + Position). Any effect of interest here met both $\mathrm{p}<.05$ and $\mathrm{BF}>3$ criteria.

\section{Results}

The overall analyses for Experiment 1 are reported in Table 1. The detailed results are shown in Fig. 1. Overall, participants performed better in trials without concurrent articulation $(M=$ $.45, S D=.27)$ relative to trials with concurrent articulation $(M$ $=.28, S D=.24)$. Participants' performance was superior for dissimilar lists $(M=.41, S D=.28)$ compared to similar lists $(M=.32, S D=.25)$. Performance was relatively similar between auditory presentation $(M=.38, S D=.26)$ and visual presentation $(M=.35, S D=.28)$. Reflecting those trends, as can be seen in Table 1, all main effects reached a conventional level of significance except the main effect of modality. Between the factors of interest (similarity, articulation, modality), only the interaction between modality and articulation reached significance. The interaction reflects the larger detrimental effect of concurrent articulation on visual presentation relative to auditory presentation. The results from the Bayesian approach were consistent with the frequentist approach.

Of key interest to the current study, as can be seen in Fig. 1, the visual similarity effect was observed with both a visual (dissimilar: $M=.39, S D=.29$; similar: $M=.31, S D=.25$ ) and an auditory presentation (dissimilar: $M=.42, S D=.27$; similar: $M=.33, S D=.25$ ). The magnitude of the visual similarity effect was equivalent between presentation modalities. The latter was supported by strong evidence in favor of an absence of the two-way interaction between similarity and modality, 
Table 1. Analyses of variance (ANOVA) for the proportion of correct responses in Experiment 1

\begin{tabular}{|c|c|c|c|c|c|}
\hline \multirow[t]{2}{*}{ Source } & \multicolumn{4}{|l|}{ ANOVA } & \multirow[t]{2}{*}{$\mathrm{BF}_{10}$} \\
\hline & $d f$ & $F$ & $P$ & $\eta_{p}^{2}$ & \\
\hline Similarity & 1,39 & 45.06 & $<.001$ & .54 & $6.75 e+26$ \\
\hline Modality & 1,39 & 2.61 & .114 & .06 & 1.82 \\
\hline Articulation & 1,39 & 150.97 & $<.001$ & .80 & $\begin{array}{r}7.69 \mathrm{e}+ \\
107\end{array}$ \\
\hline Position & $3.49,135.90$ & 172.00 & $<.001$ & .82 & $\operatorname{Inf}$ \\
\hline Similarity $\times$ Modality & 1,39 & 0.32 & .576 & .01 & $4.10 \mathrm{e}-02$ \\
\hline Similarity $\times$ Articulation & 1,39 & 2.97 & .093 & .07 & 0.88 \\
\hline Modality $\times$ Articulation & 1,39 & 23.61 & $<.001$ & .38 & $1.06 \mathrm{e}+10$ \\
\hline Similarity $\times$ Position & 5,195 & 1.33 & .251 & .03 & 0.00 \\
\hline Modality $\times$ Position & $3.37,131.45$ & 10.02 & $<.001$ & .20 & $3.04 \mathrm{e}+08$ \\
\hline Articulation $\times$ Position & $3.79,147.98$ & 15.71 & $<.001$ & .29 & $5.52 \mathrm{e}+12$ \\
\hline Similarity $\times$ Modality $\times$ Articulation & 1,39 & 0.04 & .851 & .00 & $3.70 \mathrm{e}-02$ \\
\hline Similarity $\times$ Modality $\times$ Position & $3.96,154.25$ & 1.37 & .239 & .03 & 0.00 \\
\hline Similarity $\times$ Articulation $\times$ Position & $3.84,149.65$ & 1.41 & .224 & .04 & 0.00 \\
\hline Modality $\times$ Articulation $\times$ Position & 5,195 & 1.18 & .322 & .03 & 0.00 \\
\hline Similarity $\times$ Modality $\times$ Articulation $\times$ Position & 5,195 & 0.91 & .473 & .02 & $1.00 \mathrm{e}-03$ \\
\hline
\end{tabular}

Note. The non-integer for the degree of freedom indicates that the Mauchly's test of sphericity reveals that the assumption of sphericity was violated $p$ $<.05$ and the Greenhouse-Geisser correction was applied. $\mathrm{BF}_{10}$ represents the Bayes factor in favor of the alternative model. Main-effect models were compared to the intercept-only model (Subject) and interaction models were compared to the main-effects model (Similarity + Modality + Articulation + Position). Values above 1 represent evidence in favor of the alternative model and value below 1 represent evidence in favor of the denominator model

$\mathrm{BF}_{01}=24.39$. Furthermore, the magnitude of the visual similarity effect was equivalent between modality with and without concurrent articulation, as supported by strong evidence in favor of the absence of a three-way interaction between similarity, modality, and articulation, $\mathrm{BF}_{01}=27.03$. The magnitude of the visual similarity effect was similar with or without concurrent articulation, as supported by the absence of a twoway interaction between visual similarity and articulation. Only superficial evidence was found in favor of the latter argument, $\mathrm{BF}_{01}=1.14$, probably due to a floor effect under concurrent articulation for visual stimuli, as can be seen in Fig. 1. However, when the analysis was carried out on the first three serial positions (serial position above floor performance), strong evidence was found, $\mathrm{BF}_{01}=20.41$.

\section{Discussion}

Overall, the results of the current experiment highlight the visual similarity effect for acoustic materials, indicating that visual codes can be generated for information that is not presented visually in short-term/working memory. Further, the visual similarity effect was observed with both a visual and an auditory presentation with and without concurrent articulation, and the magnitude of the visual similarity effects was equivalent between both presentation modalities. If visual information was only used as a backup modality, the visual similarity effect should have been greatly increased by articulatory suppression, but that was not the case.

Given the novelty of this demonstration, we attempted to replicate our current findings in Experiment 2 with a different set of stimuli to solidify our findings.

\section{Experiment 2}

Experiment 2 was designed to replicate our finding with a new set of stimuli. The reason this is critical is that it provides strength to our findings and reduces the likelihood that unknown, idiosyncratic features of the stimuli cause an unusual pattern of results (see, e.g., Caplan et al., 1992; Guitard et al., 2019; Lovatt et al., 2000; Neath et al., 2003). Therefore, Experiment 2 aimed at further exploring if visual codes support the memory representation of both visual and auditory word lists in immediate recall.

\section{Method}

Participants Forty different undergraduate students from the University of Missouri volunteered to participate for course credits. The mean age of the participants was 18.73 years $(S D$ $=1.13$, range $18-23) ; 18$ self-identified as a female and 22 as a 


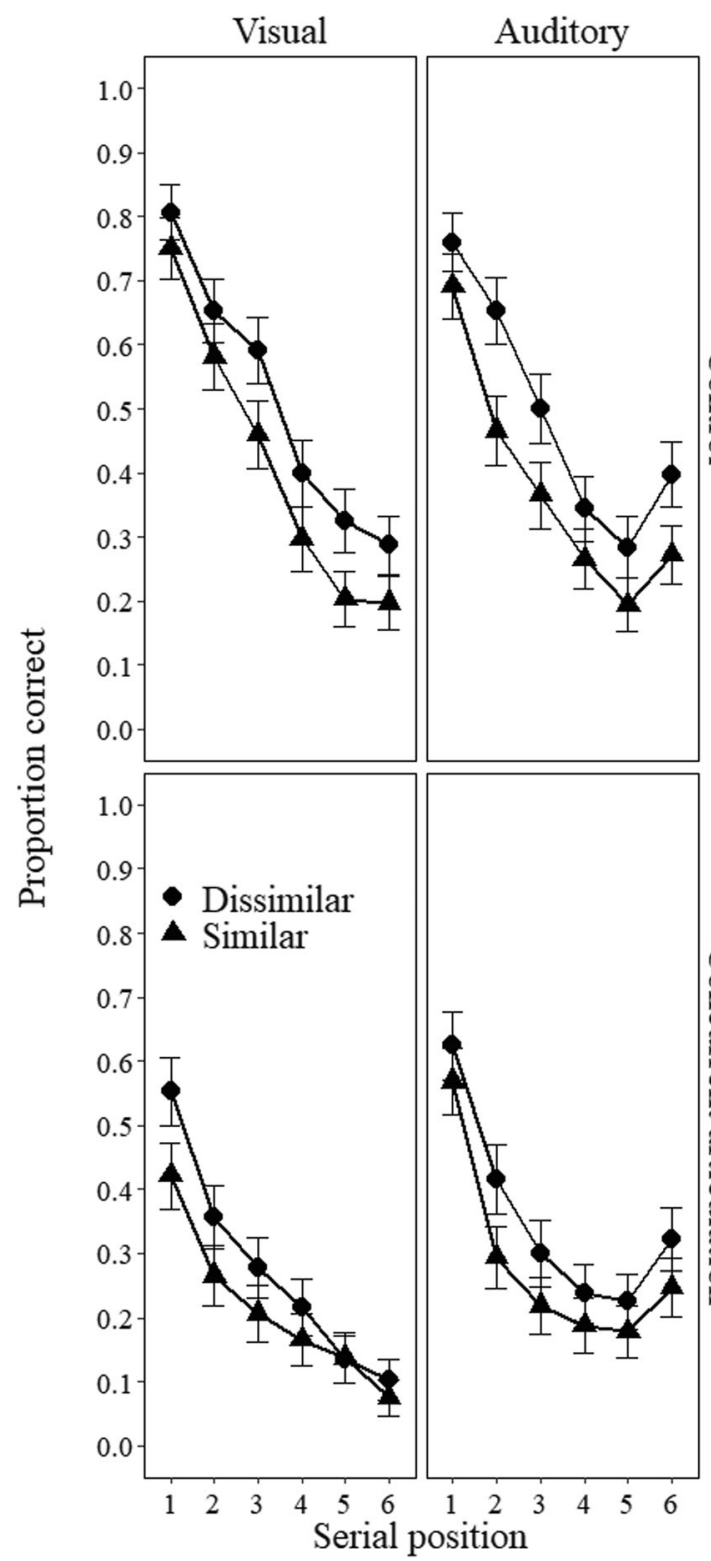

Fig. 1 Proportion of correct response for Experiment 1 as a function of visual similarity (graph parameter: dissimilar, similar), presentation modality (top labels: visual, auditory), and articulation (right side labels: control, concurrent articulation). Left two columns, Serial positions 1-6;

male. All participants were native speakers of English. The rationale for the sample size was identical to Experiment 1.

Materials A new set of stimuli was created with the same constrains as in Experiment 1 . The visually dissimilar words were DAWN, LORE, MAST, SELL, SCUM, and STAG. The visually similar words were COPE, CUFF, CURE, DUKE, GOLF, and PORE. Like Experiment 1, using the data of Simpson et al. (2013), we computed a visual similarity score for each word as was done in Chubala et al. (2020). Based on this measure, the visual similarity score was lower for

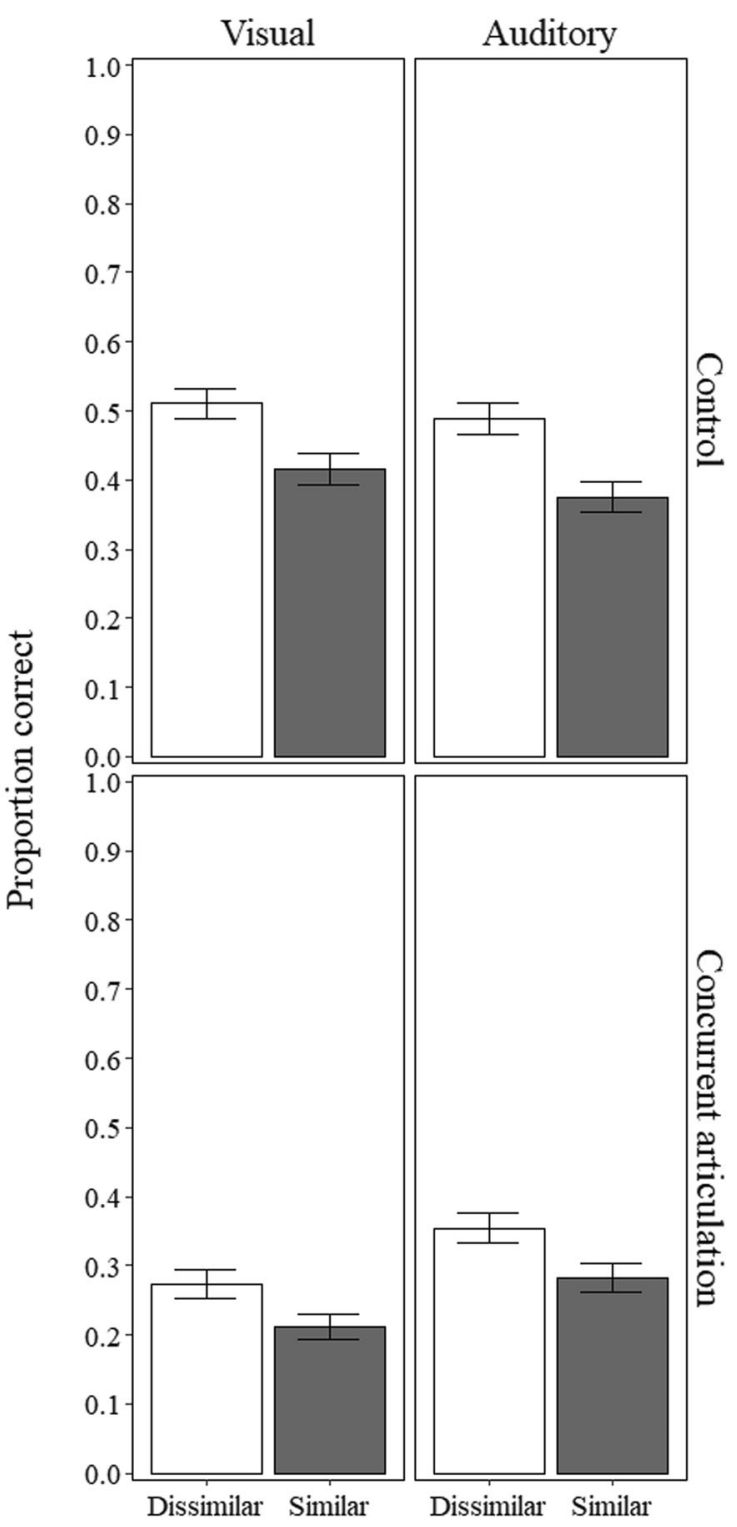

right two columns, results averaged across serial positions. Error bars represent $95 \%$ within-participant confidence intervals computed according to Morey's (2008) procedure

dissimilar lists $(M=8.32, S D=2.40)$ relative to similar lists $(M=18.60, S D=2.89), t(10)=14.51, p<0.001$. The stimuli were equated on phonological similarity using a measure based on PSIMETRICA (Mueller, Seymour, Kieras, \& Meyer, 2003). Using standard American pronunciation, the mean phonological dissimilarity scores were equivalent between the visually similar list $(M=0.25, S D=0.04)$ and the visually dissimilar list $(M=0.25, S D=0.02), t(10)=0.20, p=$ 0.84 . The stimuli were also controlled for several other dimensions such as word frequency and neighborhood size (see Table 2). 
Table 2. Descriptive statistics for the six visually similar words and the six visually dissimilar words used in Experiment 2 CnC.M LgWF LgCD LgHAL OLD OLDF PLD PLDF Length NPhon NSyll Celex Orth OrthZ OrthF

\begin{tabular}{|c|c|c|c|c|c|c|c|c|c|c|c|c|c|c|c|}
\hline \multicolumn{16}{|c|}{ Visually similar words } \\
\hline COPE & 1.78 & 2.22 & 2.18 & 8.24 & 1 & 8.64 & 1 & 8.57 & 4 & 3 & 1 & 34.51 & 19 & 1.86 & 63.15 \\
\hline CUFF & 4.61 & 2.47 & 2.38 & 6.73 & 1.7 & 7.34 & 1.15 & 7.94 & 4 & 3 & 1 & 3.75 & 8 & -0.14 & 1.17 \\
\hline CURE & 2.41 & 3.03 & 2.8 & 9.05 & 1.15 & 7.99 & 1.7 & 8.31 & 4 & 4 & 1 & 22.07 & 12 & 0.59 & 46.38 \\
\hline DUKE & 4 & 3.11 & 2.49 & 9.4 & 1.55 & 8.11 & 1 & 9.13 & 4 & 3 & 1 & 38.97 & 6 & -0.5 & 0.93 \\
\hline GOLF & 4.52 & 3.11 & 2.78 & 9.25 & 1.8 & 8.23 & 1.9 & 7.48 & 4 & 4 & 1 & 29.81 & 4 & -0.87 & 28.5 \\
\hline PORE & 4.42 & 1.4 & 1.3 & 6.03 & 1 & 8.08 & 1 & 9.82 & 4 & 3 & 1 & 0.59 & 21 & 2.22 & 128.87 \\
\hline Mean & 3.62 & 2.56 & 2.32 & 8.12 & 1.37 & 8.07 & 1.29 & 8.54 & 4 & 3.33 & 1 & 21.62 & 11.67 & 0.53 & 44.83 \\
\hline SD & 1.22 & 0.68 & 0.55 & 1.42 & 0.36 & 0.42 & 0.4 & 0.84 & 0 & 0.52 & 0 & 16.1 & 7 & 1.27 & 47.96 \\
\hline Min & 1.78 & 1.4 & 1.3 & 6.03 & 1 & 7.34 & 1 & 7.48 & 4 & 3 & 1 & 0.59 & 4 & -0.87 & 0.93 \\
\hline Max & 4.61 & 3.11 & 2.8 & 9.4 & 1.8 & 8.64 & 1.9 & 9.82 & 4 & 4 & 1 & 38.97 & 21 & 2.22 & 128.87 \\
\hline$t(10)$ & 0.44 & -0.18 & -0.06 & 0.17 & -0.26 & 0.34 & -0.97 & 0.84 & 0.00 & 0.54 & 0.00 & -0.46 & 0.00 & 0.00 & 1.90 \\
\hline$p$ & .673 & .863 & .955 & .873 & .799 & .740 & .355 & .423 & 1.00 & .599 & 1.00 & .655 & 1.00 & 1.00 & .086 \\
\hline \multicolumn{16}{|c|}{ Visually dissimilar words } \\
\hline DAWN & 4.46 & 3.11 & 2.84 & 9.02 & 1.5 & 8.17 & 1 & 8.35 & 4 & 3 & 1 & 34.92 & 8 & -0.14 & 161.72 \\
\hline LORE & 2.35 & 1.54 & 1.46 & 8.26 & 1 & 8.31 & 1 & 10.1 & 4 & 3 & 1 & 2.68 & 22 & 2.41 & 147.27 \\
\hline MAST & 4.92 & 1.92 & 1.76 & 7.39 & 1.05 & 8.68 & 1 & 9.73 & 4 & 4 & 1 & 2.68 & 18 & 1.68 & 196.46 \\
\hline SCUM & 3.9 & 2.7 & 2.59 & 8.04 & 1.8 & 7.36 & 1.35 & 8.12 & 4 & 4 & 1 & 3.03 & 4 & -0.87 & 1.64 \\
\hline SELL & 3.35 & 3.67 & 3.39 & 11.26 & 1.2 & 8.56 & 1 & 10.13 & 4 & 3 & 1 & 52.23 & 11 & 0.41 & 167.34 \\
\hline STAG & 4.39 & 1.91 & 1.76 & 5.67 & 1.35 & 7.85 & 1.35 & 7.65 & 4 & 4 & 1 & 3.69 & 7 & -0.32 & 31.31 \\
\hline Mean & 3.9 & 2.48 & 2.3 & 8.27 & 1.32 & 8.16 & 1.12 & 9.01 & 4 & 3.5 & 1 & 16.54 & 11.67 & 0.53 & 117.62 \\
\hline SD & 0.93 & 0.82 & 0.75 & 1.85 & 0.3 & 0.49 & 0.18 & 1.1 & 0 & 0.55 & 0 & 21.65 & 6.95 & 1.26 & 80.51 \\
\hline Min & 2.35 & 1.54 & 1.46 & 5.67 & 1 & 7.36 & 1 & 7.65 & 4 & 3 & 1 & 2.68 & 4 & -0.87 & 1.64 \\
\hline Max & 4.92 & 3.67 & 3.39 & 11.26 & 1.8 & 8.68 & 1.35 & 10.13 & 4 & 4 & 1 & 52.23 & 22 & 2.41 & 196.46 \\
\hline
\end{tabular}

Note. Brysbaert et al. (2014): CnC.M = concreteness; Brysbaert \& New (2009): LgWF = Log word frequency; $\operatorname{LgCD}=\log$ contextual diversity from SUBTLEXUS; Balota et al. (2007): LgHal = log HAL frequency; OLD = orthographic Levenshtein distance; OLDF = frequency of orthographic Levenshtein neighbors; PLD= phonological Levenshtein distance; OLDF=frequency of phonological Levenshtein neighbors; Length= number of letters; NPhon = number of phonemes; NSYll = number of syllables; Medler \& Binder (2005): Celex = Celex frequency; Orth = number of orthographic neighbors; OrthZ = z-transformed ORTH; OrthF = frequency of orthographic neighbors

Design, procedure, and data analysis The design, procedure, and data analysis were identical to Experiment 1, except that a different set of words was used.

\section{Results}

The analyses for Experiment 2 are reported in Table 3. The detailed results are shown in Fig. 2. Like Experiment 1, participants performed better in trials without concurrent articulation $(M=.50, S D=.27)$ relative to trials with concurrent articulation $(M=.34, S D=.25)$. Participants performed better for dissimilar lists $(M=.45, S D=.28)$ compared to similar lists $(M=.40, S D=.26)$. Participants' performance was similar between the auditory presentation $(M=.42, S D=.27)$ and the visual presentation $(M=.42, S D=.28)$. As in the results of Experiment 1, all main effects reached conventional levels of significance except the main effect of modality. For the interaction between the factors of interest (similarity, articulation, modality), like Experiment 1, only the interaction between modality and articulation reached significance. The latter interaction reflected the more disruptive effect of concurrent articulation on the recall in the visual presentation condition relative to the auditory presentation condition. The results from the Bayesian approach for the aforementioned effects were consistent with the frequentist approach.

Of particular theoretical interest, as was the case in Experiment 1, the visual similarity effect was observed with both a visual (dissimilar: $M=.45, S D=.29$; similar: $M=.40, S D=.27$ ) and an auditory presentation (dissimilar: $M=.45, S D=.28$; similar: $M=.39, S D=$ .26). Again, the magnitude of the visual similarity effect was equivalent between presentation modalities, as supported by strong evidence, $\mathrm{BF}_{01}=40.00$, in favor of an absence of the two-way interaction between similarity and modality. Supporting the equivalent magnitude of the visual similarity effect between modality and 
Table 3 Analyses of variance (ANOVA) for the proportion of correct responses in Experiment 2

\begin{tabular}{|c|c|c|c|c|c|}
\hline \multirow[t]{2}{*}{ Source } & \multicolumn{4}{|l|}{ ANOVA } & \multirow[t]{2}{*}{$\mathrm{BF}_{10}$} \\
\hline & $d f$ & $F$ & $p$ & $\eta_{p}^{2}$ & \\
\hline Similarity & 1,39 & 16.99 & $<.001$ & .30 & $9.44 \mathrm{e}+07$ \\
\hline Modality & 1,39 & 0.01 & .945 & .00 & $1.80 \mathrm{e}-02$ \\
\hline Articulation & 1,39 & 177.24 & $<.001$ & .82 & $\begin{array}{r}3.09 \mathrm{e}+ \\
199\end{array}$ \\
\hline Position & $3.01,117.50$ & 133.54 & $<.001$ & .77 & $\operatorname{Inf}$ \\
\hline Similarity $\times$ Modality & 1,39 & 0.02 & .896 & .00 & $2.50 \mathrm{e}-02$ \\
\hline Similarity $\times$ Articulation & 1,39 & 0.32 & .577 & .01 & $3.30 \mathrm{e}-02$ \\
\hline Modality $\times$ Articulation & 1,39 & 27.87 & $<.001$ & .42 & $1.50 \mathrm{e}+06$ \\
\hline Similarity $\times$ Position & 5,195 & 1.78 & .120 & .04 & 0.00 \\
\hline Modality $\times$ Position & $3.79,147.97$ & 18.69 & $<.001$ & .32 & $3.91 \mathrm{e}+14$ \\
\hline Articulation $\times$ Position & $3.19,124.33$ & 9.26 & $<.001$ & .19 & $2.05 \mathrm{e}+05$ \\
\hline Similarity $\times$ Modality $\times$ Articulation & 1,39 & 0.04 & .844 & .00 & $3.70 \mathrm{e}-02$ \\
\hline Similarity $\times$ Modality $\times$ Position & $3.70,144.09$ & 2.88 & .016 & .07 & $1.10 \mathrm{e}-02$ \\
\hline Similarity $\times$ Articulation $\times$ Position & $4.07,158.52$ & 0.47 & .799 & .01 & 0.00 \\
\hline Modality $\times$ Articulation $\times$ Position & $3.99,155.71$ & 2.85 & .017 & .07 & $3.00 \mathrm{e}-03$ \\
\hline Similarity $\times$ Modality $\times$ Articulation $\times$ Position & $4.07,158.87$ & 0.80 & .548 & .02 & 0.00 \\
\hline
\end{tabular}

Note. The non-integer for the degree of freedom indicates that the Mauchly's test of sphericity reveals that the assumption of sphericity was violated $p$ $<.05$ and the Greenhouse-Geisser correction was applied. $\mathrm{BF}_{10}$ represents the Bayes factor in favor of the alternative model. Main-effect models were compared to the intercept-only model (Subject) and interaction models were compared to the main-effects model (Similarity + Modality + Articulation + Position). Values above 1 represent evidence in favor of the alternative model and value below 1 represent evidence in favor of the denominator model

articulation, strong evidence was found in favor of the absence of a three-way interaction between similarity, modality, and articulation, $\mathrm{BF}_{01}=27.03$. Furthermore, the magnitude of the visual similarity effect was similar with or without concurrent articulation, a point supported by strong evidence, $\mathrm{BF}_{01}=30.30$, in favor of the absence of a two-way interaction between visual similarity and articulation.

\section{Discussion}

Overall, the results of the current experiment were consistent with the results of Experiment 1. This successful replication with a different set of stimuli solidifies our findings and previous conclusion. Most importantly, the visual similarity effect in the auditory condition once more supports the notion that visual codes can be generated for information that is not presented visually in short-term/working memory. In Experiment 2, as in Experiment 1, the visual similarity effect was observed with both presentation modalities and in the presence or absence of concurrent articulation during the presentation. Moreover, the magnitude of the visual similarity effects was equivalent between both presentation modalities and between articulation conditions.

\section{Cross-experiment analysis}

Given that presentation modality was blocked and counterbalanced, for each experiment half the participants started with a visual presentation and the other half started with an auditory presentation. In this section, we further examine our current results to rule out an alternative account. More specifically, one alternative account of our current finding is that participants who started with a visual presentation might be biased toward using a visual representation of the auditory stimuli. In other words, the visual similarity effect would be attributed to a carryover effect from the processing of visual materials to the auditory case. If this alternative account is correct, we should not observe a visual similarity effect with an auditory presentation when participants started with an auditory block. To test this alternative view, in this section we examine the performance of the 40 participants, 20 in each experiment, in the auditory condition presented as the first block. We conducted a cross-experiments analysis because power analyses suggested that the first trial block within each experiment alone did not have sufficient power to detect the critical comparison.

Proportion correct was analyzed as a function of the experiment, visual similarity, concurrent articulation, and serial position. The results of the cross-experiment analysis are reported in Table 4. The detailed results are shown in Fig. 3. 


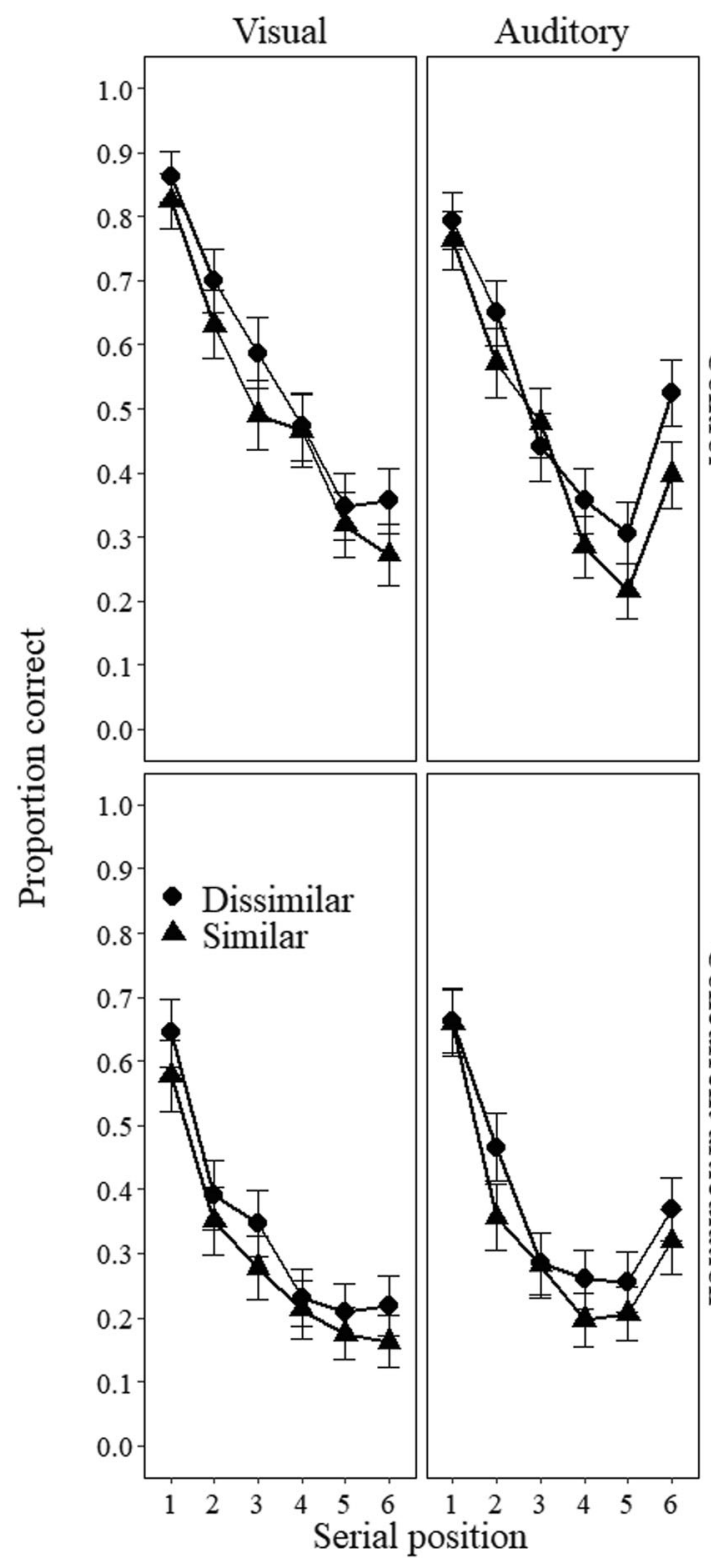

Fig. 2 Proportion of correct response for Experiment 2 as a function of visual similarity (graph parameter: dissimilar, similar), presentation modality (top labels: visual, auditory), and articulation (right side labels: control, concurrent articulation). Left two columns, Serial positions $1 \times 6$;

Consistent with our overall results and ruling out this alternative account, a visual similarity effect was observed with an auditory presentation in the first block (dissimilar: $M=$ $.36, S D=.24$; similar: $M=.32, S D=.23), \mathrm{BF}_{10}=$ 85.52. The results of the analysis also reveal that all main effects reached conventional levels of significance except for the main effect of the experiment. For the interactions, none of the interactions between similarity, articulation, and experiment reached significance. However, of theoretical importance to the current study, once more the magnitude of the visual similarity effect was similar with (dissimilar: $M=.42, S D=.24$; similar:

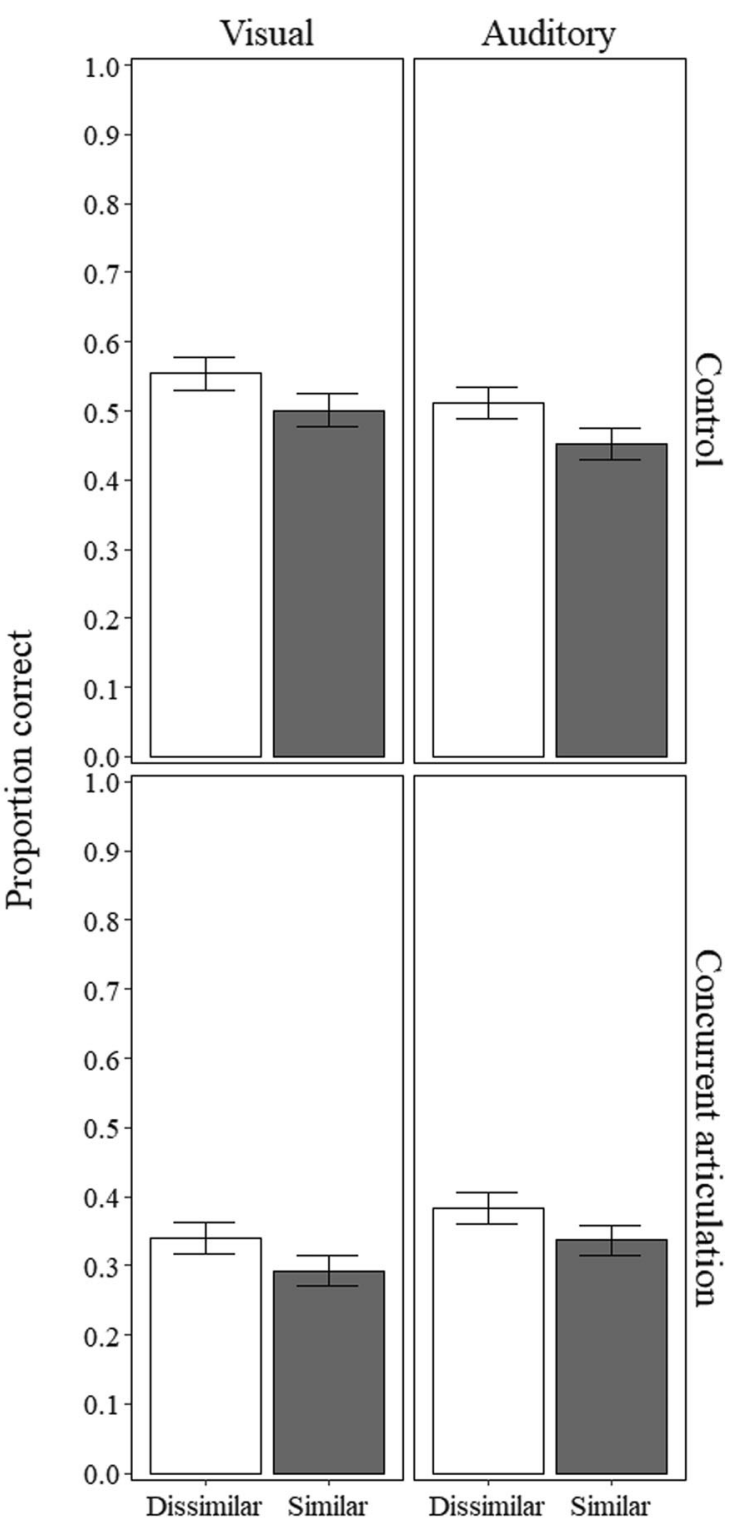

right two columns, results averaged across serial positions. Error bars represent $95 \%$ within-participant confidence intervals computed according to Morey's (2008) procedure

$M=.38, S D=.25)$ or without concurrent articulation (dissimilar: $M=.29, S D=.22$; similar: $M=.26, S D=$ $.20)$, a point supported by strong evidence, $\mathrm{BF}_{01}=$ 23.26, in favor of the absence of a two-way interaction between visual similarity and articulation. This latter pattern of results was also observed in both experiments, as supported by positive evidence, $\mathrm{BF}_{01}=7.04$, in favor of an absence of the three-way interaction between similarity, articulation, and experiment.

In sum, further empirical exploration of our data revealed that the visual similarity effect with an auditory presentation occurs despite the fact that participants started with the 
Table 4 Analyses of variance (ANOVA) for audio first block on the proportion of correct responses in Experiment 1 and in Experiment 2

\begin{tabular}{|c|c|c|c|c|c|}
\hline \multirow[t]{2}{*}{ Source } & \multicolumn{4}{|l|}{ ANOVA } & \multirow[t]{2}{*}{$\mathrm{BF}_{10}$} \\
\hline & $d f$ & $F$ & $p$ & $\eta_{p}^{2}$ & \\
\hline Similarity & 1,38 & 6.41 & .016 & .14 & 85.52 \\
\hline Experiment & 1,38 & 2.16 & .150 & .05 & 0.17 \\
\hline Articulation & 1,38 & 69.32 & $<.001$ & .65 & $6.94 \mathrm{e}+28$ \\
\hline Position & $3.10,117.65$ & 96.09 & $<.001$ & .72 & $\begin{array}{r}8.76 \mathrm{e}+ \\
175\end{array}$ \\
\hline Similarity $\times$ Experiment & 1,38 & 0.00 & .976 & .00 & 0.04 \\
\hline Similarity $\times$ Articulation & 1,38 & 0.16 & .693 & .00 & 0.04 \\
\hline Experiment $\times$ Articulation & 1,38 & 0.27 & .606 & .01 & 0.05 \\
\hline Similarity $\times$ Position & 5,190 & 1.72 & .131 & .04 & 0.00 \\
\hline Experiment $\times$ Position & $3.10,117.65$ & 2.36 & .074 & .06 & 0.59 \\
\hline Articulation $\times$ Position & $4.13,157.00$ & 6.54 & $<.001$ & .15 & 1.60 \\
\hline Similarity $\times$ Experiment $\times$ Articulation & 1,38 & 1.02 & .318 & .03 & 0.14 \\
\hline Similarity $\times$ Experiment $\times$ Position & 5,190 & 1.22 & .302 & .03 & 0.00 \\
\hline Similarity $\times$ Articulation $\times$ Position & 5,190 & 0.94 & .458 & .02 & 0.00 \\
\hline Experiment $\times$ Articulation $\times$ Position & $4.13,157.00$ & 1.09 & .363 & .03 & 0.00 \\
\hline Similarity $\times$ Experiment $\times$ Articulation $\times$ Position & 5,190 & 1.21 & .304 & .03 & 0.01 \\
\hline
\end{tabular}

Note. The non-integer for the degree of freedom indicates that the Mauchly's test of sphericity reveals that the assumption of sphericity was violated $p$ $<.05$ and the Greenhouse-Geisser correction was applied. $\mathrm{BF}_{10}$ represents the Bayes factor in favor of the alternative model. Main-effect models were compared to the intercept-only model (Subject) and interaction models were compared to the main-effects model (Similarity + Experiment + Articulation + Position). Values above 1 represent evidence in favor of the alternative model and value below 1 represent evidence in favor of the denominator model

auditory presentation block. Therefore, our results cannot be attributed to a carryover effect from the processing of visual materials to the auditory case.

\section{General discussion}

Over the years, researchers have devoted far less attention to visual codes relative to phonological codes in the working/ short-term memory literature (Hurlstone, Hitch, \& Baddeley, 2014). In the current study, we have addressed this imbalance by studying visual codes and focusing on a critical aspect of modern models of working memory, namely the principle that participants' responses do not depend primarily on what kinds of materials are presented, but on what kinds of code participants generated from those materials. Using the visual similarity effect, we explored whether participants generated visual codes for both visual and acoustic materials. Although visual codes for acoustic materials could be theoretically anticipated from some points of view, this assumption has remained without empirical support until this study.

In two experiments we tested this assumption by manipulating visual similarity, presentation modality, and concurrent articulation. Our results are clear and can be summarized as follows. In two experiments with two different sets of stimuli controlled for a vast number of psycholinguistic factors, we observed a visual similarity effect with both a visual and an auditory presentation of word lists, with and without task-irrelevant, concurrent articulation to suppress the conversion of words to a phonological form.

The visual similarity effect with visual materials adds to the growing list supporting the presence of visual codes for visual materials (Avons \& Mason, 1999; Chubala, et al., 2020; Logie et al., 2000; Logie et al., 2016; Poirier et al., 2007; Saito et al., 2008; Smyth et al., 2005). Importantly, the magnitude of the visual similarity effect was equivalent between presentation modalities in both experiments. Furthermore, when removing the last three serial positions near floor performance in Experiment 1, both Experiment 1 and Experiment 2 provide strong support in favor of the equivalent magnitude of the visual similarity effect with or without concurrent articulation. Therefore, our results are convergent with previous demonstrations that show independence between visual similarity and concurrent articulation (see, e.g., Logie et al., 2000; Poirier et al., 2007). Further analysis also reveals that these conclusions remain valid when participants started with the auditory presentation block for both experiments, ruling out a visual similarity effect based only on a carryover effect from the processing of visual materials to the auditory case.

Our results confirm with visual codes an important aspect of the modern model of working memory, that participants' responses do not depend primarily on what kinds of materials are presented, but on what kinds of code participants generated from those materials. For the first time, we were able to 


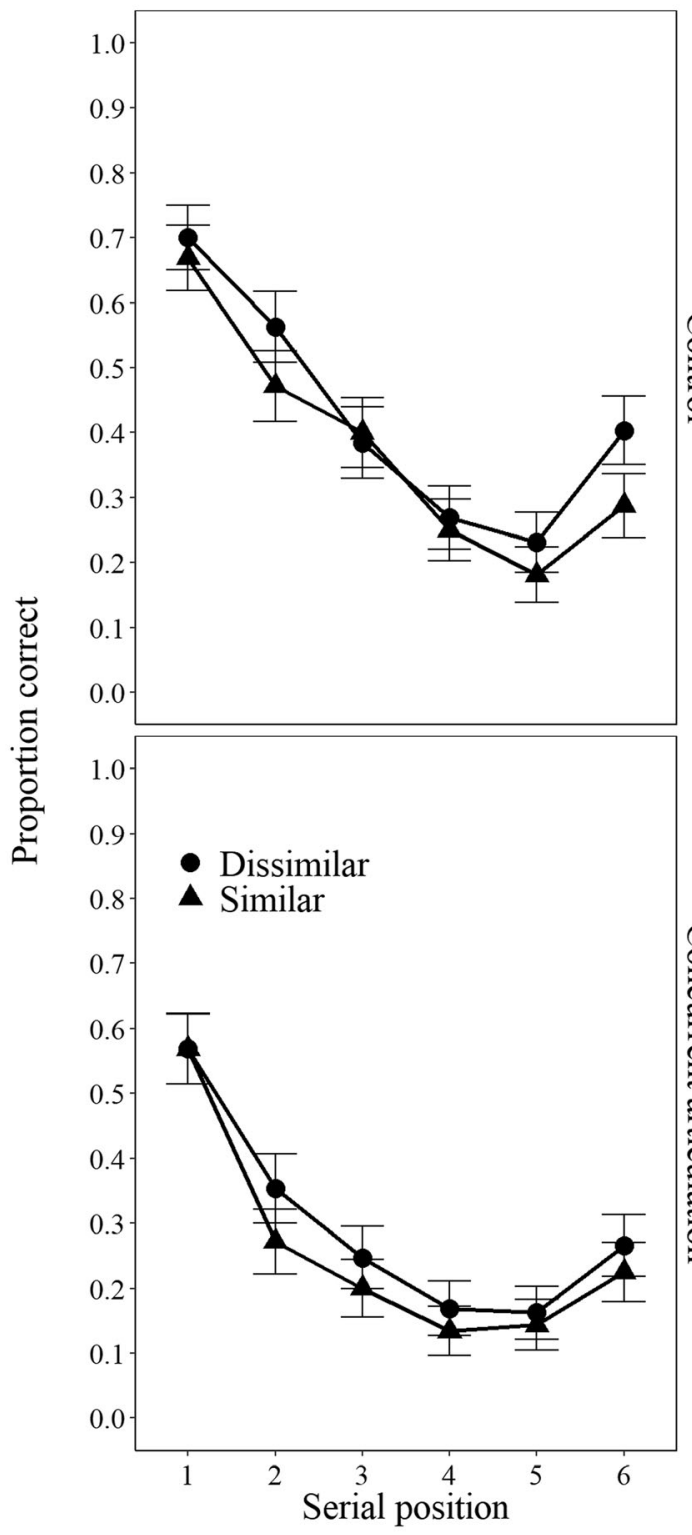

Fig. 3 Proportion of correct response for Experiment 1 and Experiment 2 auditory first block as a function of visual similarity (graph parameter: dissimilar, similar) and articulation (right side labels: control, concurrent articulation). Left column, Serial positions 1-6; right column, results

demonstrate that participants were able to generate visual codes for both visual and acoustic material. The utilization of visual codes was demonstrated by observing a better performance for visually dissimilar words relative to visually similar words in both experiments.

If visual codes were not used, we would have observed similar performance on lists sharing some visual features to the performance on lists not sharing these features. This is the case inasmuch as we selected stimuli in a manner that painstakingly controlled for various factors that could have been confounded with visual similarity. Our findings further

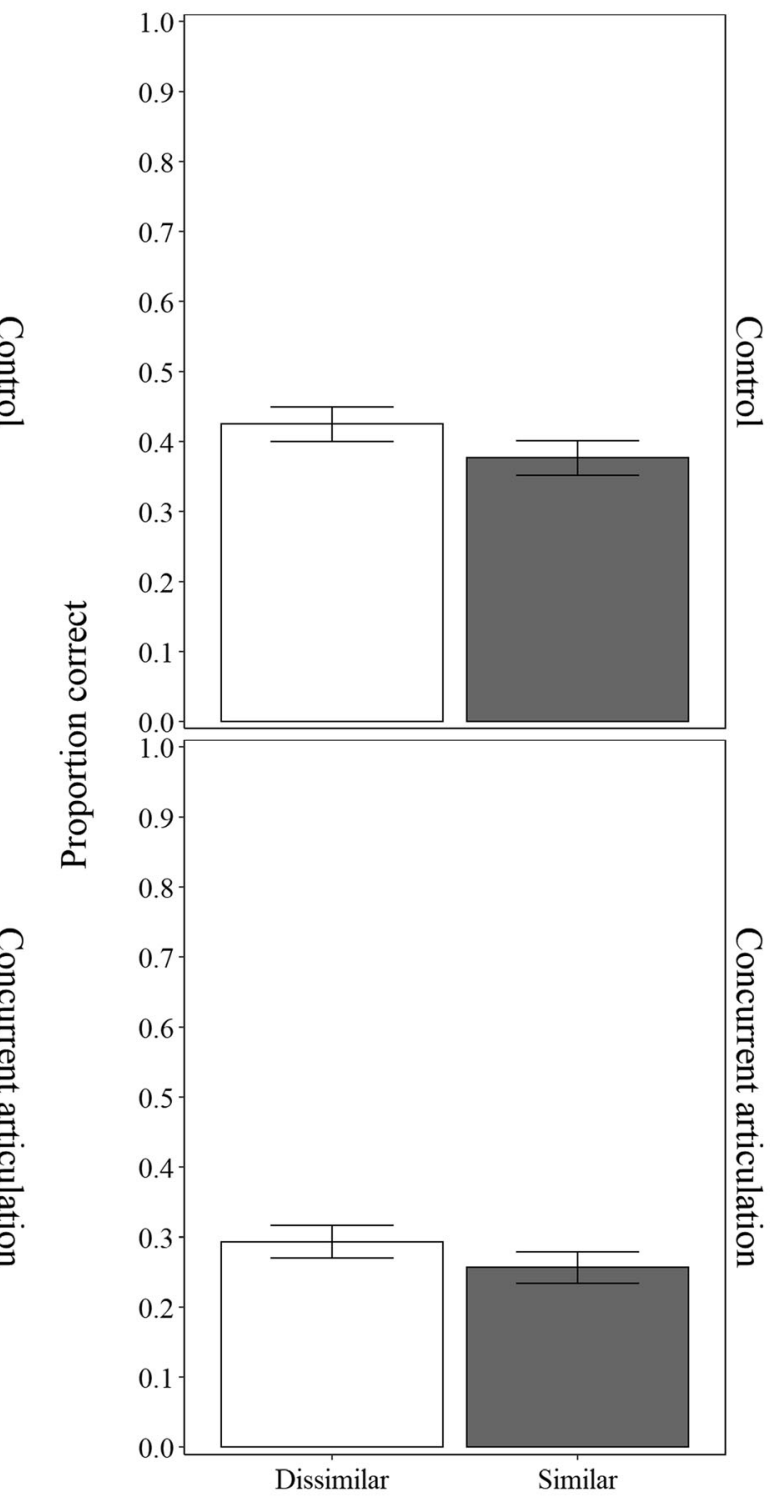

averaged across serial positions. Error bars represent $95 \%$ withinparticipant confidence intervals computed according to Morey's (2008) procedure

support the major impetus of studying similarity to better understand the nature of memory representation and the notion that features need not be intrinsic to a stimulus, but can be features added by the perceiver, as was the case for visual codes in the auditory materials condition.

Consistent with the pioneering work of Conrad (1964), who showed that visual material gives rise to an internal acoustic code, we found that acoustic material gives rise to an internal visual orthographic code. One major implication for modern theories of memory is that we can rule out an asymmetrical prediction in which conversion only goes from 
a secondary modality for language (vision, orthography) to a primary modality (acoustics, phonology) and not in the reverse direction.

Other aspects of the present results, namely the results under concurrent articulation, also have important implications that may help to refine theories of memory. According to one possible theoretical view, as reported previously by Hitch et al. (1989) and Poirier et al. (2007), visual information would serve as a backup to phonological codes when phonological information is not sufficient. Based on such a theoretical view, the effect of visual similarity should be larger with concurrent articulation than without it, as concurrent articulation should suppress the phonological code (at least in the visual modality). This was not the case in our results, in which there was strong evidence that similarity did not interact with articulation, suggesting a need to rethink this supposition in these theories.

Some models seem to anticipate this absence of an interaction of similarity with articulation. In models focusing on many concurrent, activated features, such as the Feature Model (Chubala et al., 2020; Nairne, 1990) and the embedded processes model (Cowan, 1988, 2019), both phonological and orthographic features of stimuli could be generated amodally, resulting in overwriting interference between similar features of different words regardless of their presentation modality. We believe that the prediction would be that concurrent articulation should interfere with phonological codes but should have limited to no effect on the use of visual codes, which could be used independent of phonological codes regardless of the presentation modality. This treatment of phonological and orthographic, amodal features as different from one another is consistent with a recent implementation of the Feature Model in which a new parameter stood for the separation of different features of these sorts that are processed separately (Poirier et al., 2019).

The absence of an interaction between visual similarity and articulation could also be anticipated within the primacy model of Page and Norris (1998). In that model, it is easy enough to obtain visual similarity effects in a manner comparable to phonological similarity effects (Logie et al., 2016). The model posits a two-stage process in which a stage with decaying activation of items is followed by an output stage in which items having similar features can be confused with one another. In that model, the role of concurrent articulation was said to be to suppress rehearsal of items, and the effect of rehearsal was described within the discussion of the first stage. Thus, Page and Norris (p. 773, Fig. 11) describe the first stage as "insensitive to item similarity" and the second stage as "effectively transparent to nonconfusable items." With that description, therefore, concurrent articulation and any visual similarity (in the extension by Logie et al.) operate on different stages and might therefore be expected not to interact. Thus, in sum, although the present data do not rule out entire classes of models, they are useful in validating and constraining some extant models.

Another consistent result across experiments was a larger detrimental effect of concurrent articulation when introduced during encoding for visually presented information relative to auditory presentation. The traditional interpretation of this effect is that with concurrent articulation occupying phonological processing, a phonological code in working memory is not well formed for printed materials, whereas spoken materials result in a phonological code more automatically, even during concurrent articulation (Baddeley, Lewis, \& Vallar, 1984; Cowan, Cartwright, Winterowd, \& Sherk, 1987; Peterson \& Johnson, 1971). It is comforting to find traditional effects like this, as it diminishes the possibility that something about our stimuli was out of the ordinary and that some kind of irregularity produced the visual similarity effect with acoustic materials.

\section{Future directions}

This study is an initial step to better understanding the complexity of memory representation in working/short-term memory. For years, many researchers thought that this memory representation was predominantly based on phonological codes. However, due to studies like this one (e.g., Logie et al., 1996; Logie, 2018; Morrison et al., 2016), we now know that many codes, such as semantic, visual, and spatial codes, also support memory representations. We encourage researchers to further study visual codes, as very little is known compared to phonological codes. One interesting line of research might be to better understand the relation between phonological codes and visual codes for both presentation modalities. Better understanding of the interplay between visual and phonological codes would provide further constraints on modern models of memory. For those who wish to research visual codes, we recommend controlling for as many psycholinguistic factors as possible, using different sets of stimuli and exploring response modalities. For instance, different results might be obtained with a spoken response modality.

There might be important factors that we have overlooked, such as output time, which has been an important factor in the memory literature, particularly in the syllable-based word length effect (see, e.g., Cowan, Day, Saults, Keller, Johnson, \& Flores, 1992). However, this particular concern seems unlikely to be critical, inasmuch as the visual similarity effect was observed by Chubala et al. (2020) using a serial recognition of order task. One issue warranting further examination is that, contrary to some manipulations of visual similarity of orthography (see, e.g., Logie et al., 2000), the present stimuli were generated with a measure focusing on inter-letter similarity rather than inter-word similarity. It would be important for future studies to explore if both inter-word and inter-letter similarity yield similar patterns of results with auditory 
presentation. Similar patterns of results were observed with a visual presentation in Chubala et al., and we therefore expect that both inter-word and inter-letter similarity would yield similar patterns of results with an auditory presentation.

\section{Conclusion}

In sum, we have tested an important principle that may underlie working memory models, which is that responses do not depend primarily on the materials presented, but on the codes generated from those materials. We have provided further support for this assumption with visual codes, which have received far too little attention in the working/short-term memory literature. Using the visual similarity effect, we have successfully demonstrated for the first time that visual codes are generated for not only visual but also auditory verbal materials. We have shown that the magnitude of the visual similarity effect in serial recall was similar between modalities, and with and without concurrent articulation, suggesting that visual codes are not limited to a backup modality for when phonological codes are missing. In conclusion, we believe that many models of verbal working memory have oversimplified how information is represented, due to insufficient attention to visual codes and, as further research may show, other types of non-phonological codes.

Author Note We thank Leonie M. Miller for assistance with calculating the PSIMETRICA measures. Thanks are also due to Bret A. Glass, Maximilian Stroyeck, and Caleb Burns for assistance with data collection. This research was supported by NIH Grant R01-21338 to Nelson Cowan. While working on this manuscript, Dominic Guitard was supported by a graduate scholarship from NSERC.

Open Practices Statement The materials are included in this manuscript. The data for all experiments are available on the Open Science Framework and will be made public upon publication (https://osf.io/ngpe9/?view_only= 3866f7ca6b9148d189ddd80bb55e68a3).

\section{References}

Avons, S. E., \& Mason, A. (1999). Effects of visual similarity on serial report and item recognition. Quarterly Journal of Experimental Psychology, 52A, 217-240.

Ayres, T. J., Jonides, J., Reilman, J. S., Egan, I. C, \& Howard, D. (1979). Differing suffix effects for the same physical stimulus. Journal of Experimental Psychology: Human Learning and Memory, 5, 315321.

Baddeley, A. D. (1966). Short-term memory for word sequences as a function of acoustic, semantic, and formal similarity. Quarterly Journal of Experimental Psychology, 18A, 362-365.
Baddeley, A. D., \& Hitch, G. J. (1974). Working memory. In G. H. Bower (Ed.), Recent Advances in Learning and Motivation Vol. VIII (pp. 47-90). New York: Academic Press.

Baddeley, A. D., Lewis, V. J., \& Vallar, G. (1984). Exploring the articulatory loop. Quarterly Journal of Experimental Psychology, 36, 233-252.

Balota, D. A., Yap, M. J., Cortese, M. J., Hutchison, K. A., Kessler, B., Loftis, B., Neely, J. H., Nelson, D. L., Simpson, G. B., \& Treiman, R. (2007). The English Lexicon Project. Behavior Research Methods, 39, 445-459.

Brysbaert, M., \& New, B. (2009). Moving beyond Kučera and Francis: A critical evaluation of current word frequency norms and the introduction of a new and improved word frequency measure for American English. Behavior Research Methods, 41, 977-900.

Brysbaert, M., Warriner, A. B., \& Kuperman, V. (2014). Concreteness ratings for 40 thousand generally known English word lemmas. Behavior Research Methods, 46, 904-911.

Burgess, N., \& Hitch, G. J. (1999). Memory for serial order: A network model of the phonological loop and its timing. Psychological Review, 106, 551-581.

Caplan, D., Rochon, E., \& Waters, G. S. (1992). Articulatory and phonological determinants of word length effects in in span tasks. The Quarterly Journal of Experimental Psychology Section A, 45, 177192.

Chubala, C. M., Guitard, D., Neath, I., Saint-Aubin, J., \& Surprenant, A. M. (2020). Visual similarity effects in immediate serial recall and (sometimes) in immediate serial recognition. Memory \& Cognition. 48, 411-425.

Coltheart, V. (1993). Effects of phonological similarity and concurrent irrelevant articulation on short-term-memory recall of repeated and novel word lists. Memory \& Cognition, 21, 539-545.

Conrad, R. (1964). Acoustic confusions in immediate memory. British Journal of Psychology, 55, 75-84.

Conrad, R., \& Hull, A. J. (1964). Information, acoustic confusion and memory span. British Journal of Psychology, 55, 429-432.

Cowan, N. (1988). Evolving conceptions of memory storage, selective attention, and their mutual constraints within the human information processing system. Psychological Bulletin, 104, 163-191.

Cowan, N., Day, L., Saults, J. S., Keller, T. A., Johnson, T., \& Flores, L. (1992). The role of verbal output time in the effects of word length on immediate memory. Journal of Memory and Language, 31, 117. https://doi.org/10.1016/0749596X(92)90002-F

Cowan, N. (2019) Short-term memory based on activated long-term memory: A review in response to Norris (2017). Psychological Bulletin, 145, 822-847.

Cowan, N., Cartwright, C., Winterowd, C., \& Sherk, M. (1987). An adult model of preschool children's speech memory. Memory and Cognition, 15, 511-517.

Crowder, R. G. (1979). Similarity and serial order in memory. In G. H. Bower (Ed.), The psychology of learning and motivation Vol. 13 (pp. 319-353). New York: Academic Press.

Farrell, S., \& Lewandowsky, S. (2003). Dissimilar items benefit from phonological similarity in serial recall. Journal of Experimental Psychology: Learning, Memory \& Cognition, 29, 838-849.

Faul, F., Erdfelder, E., Buchner, A., \& Lang, A.-G. (2009). Statistical power analyses using $\mathrm{G}^{*}$ Power3.1: Tests for correlation and regression analyses. Behavior Research Methods, 41, 1149-1160.

Guitard, D., Miller, L. M., Neath, I., \& Roodenrys, S. (2019) Does contextual diversity affect serial recall? Journal of Cognitive Psychology, 31, 379-396.

Henson, R. N. A. (1998). Short-term memory for serial order: The StartEnd Model. Cognitive Psychology, 36, 73-137.

Hitch, G. J., Woodin, M. E., \& Baker, S. (1989). Visual and phonological components of working memory in children. Memory \& Cognition, $17,175-185$. 
Hurlstone, M. J., Hitch, G. J., \& Baddeley, A. D. (2014). Memory for serial order across domains: An overview of the literature and directions for future research. Psychological Bulletin, 140(No. 2), 339373.

JASP Team (2019). JASP (Version 0.11.1.0) [Computer software] https://jasp-stats.org/

Kass, R., \& Raftery, A. (1995). Bayes Factors. Journal of the American Statistical Association, 90, 773-795.

Lewandowsky, S., \& Farrell, S. (2008). Phonological similarity in serial recall: constraints on theories of memory. Journal of Memory and Language, 58, 429-448.

Logie, R.H. (2018). Human Cognition: Common Principles and Individual Variation. Journal of Applied Research in Memory and Cognition, 7, 471-486.

Logie, R.H., Della Sala, S., Laiacona, M., Chalmers, P. \& Wynn, V. (1996). Group aggregates and individual reliability: The case of verbal short-term memory. Memory \& Cognition, 24, 305-321.

Logie, R. H., Della Sala, S., Wynn, V., \& Baddeley, A.D. (2000). Visual similarity effects in immediate verbal serial recall. Quarterly Journal of Experimental Psychology, 53A, 626-646.

Logie, R. H., Saito, S., Morita, A., Varma, S., \& Norris, D. (2016). Recalling visual serial order for verbal sequences. Memory \& Cognition, 44, 590-607.

Lovatt, P., Avons, S. E., \& Masterson, J. (2000). The word length effect and disyllabic words. Quarterly Journal of Experimental Psychology, 53A, 1-22.

Medler, D. A., \& Binder, J. R. (2005). MCWord: An on-line orthographic database of the English language. Retrieved from http://www.neuro. mcw.edu/mcword/

Morey, R. D. (2008). Confidence intervals from normalized data: A correction to Cousineau (2005). Tutorial in Quantitative Methods for Psychology, 4, 61-64.

Morey, R. D. \& Rouder, J. N. (2018). BayesFactor: Computation of Bayes Factors for Common Designs. R package version 0.9.124.2. https://CRAN.R-project.org/package=BayesFactor

Morrison, A. B., Rosenbaum, G. M., Fair, D., \& Chein, J. M. (2016). Variation in strategy use across measures of verbal working memory. Memory \& Cognition, 44, 922-936.

Mueller, S. T., Seymour, T. L., Kieras, D. E., \& Meyer, D. E. (2003). Theoretical implications of articulatory duration, phonological similarity, and phonological complexity in verbal working memory. Journal of Experimental Psychology: Learning, Memory, and Cognition, 29, 1353-1380.

Nairne, J.S. (1990). A feature model of immediate memory. Memory \& Cognition, 18, 251-269.

Neath, I., Surprenant, A.M., \& Crowder, R. G. (1993). The contextdependent stimulus suffix effect. Journal of Experimental Psychology: Learning, Memory, and Cognition, 19, 698-703.

Neath, I., Bireta, T. J., \& Surprenant, A. M. (2003). The time based word length effect and stimulus set specificity. Psychonomic Bulletin \& Review, 10, 430-434.

Oberauer, K., Lewandowsky, S., Awh, E., Brown, G.D.A., Conway, A., Cowan, N., Donkin, C., Farrell, S., Hitch, G.J., Hurlstone, M., Ma, W.J., Morey, C. C., Nee, D. E., Schweppe, J., Vergauwe, E., \& Ward, G. (2018). Benchmarks for models of working memory. Psychological Bulletin, 144, 885-958.
Paivio, A. (1971). Imagery and verbal processes. Oxford, UK: Rhinehart, \& Winston. Paivio, A. (1991). Dual coding theory: Retrospect and current status. Canadian Journal of Psychology, 45, 255-287. https://doi.org/10.1037/h0084295

Paivio, A. (1991). Dual coding theory: Retrospect and current status. Canadian Journal of Psychology, 45, 255-287. https://doi.org/10. 1037/h0084295

Paivio, A. (2007). Mind and its evolution: A dual coding theoretical approach. Mahwah, NJ: Erlbaum.

Page, M. P. A., \& Norris, D. (1998). The Primacy Model: A new model of immediate serial recall. Psychological Review, 105, 761-781. https://doi.org/10.1037/0033-295X.105.4.761-781

Peterson, L. R., \& Johnson, S. T. (1971). Some effects of minimizing articulation on short-term retention. Journal of Verbal Learning \& Verbal Behavior, 10, 346-354.

Poirier, M., \& Saint-Aubin, J. (1996). Immediate serial recall, word frequency, item identity and item position. Canadian Journal of Experimental Psychology, 50, 408-412.

Poirier, M., Saint-Aubin, J., Musselwhite, K., Mohanadas, T., \& Mahammed, G. (2007). Visual similarity effects on short-term memory for order: The case of verbally labeled pictorial stimuli. Memory \& Cognition, 35, 711-723.

Poirier, M., Yearsley, J.M., Saint-Aubin, J., Fortin, C., Gallant, G., \& Guitard, D. (2019). Dissociating visuo-spatial and verbal working memory: It's all in the features. Memory \& Cognition, 47, 603-618.

R Core Team (2019). R: A language and environment for statistical computing. R Foundation for Statistical Computing, Vienna, Austria. URL https://www.R-project.org/.

Rouder, J. N., Speckman, P. L., Sun, D., Morey, R. D., \& Iverson, G. (2009). Bayesiant tests for accepting and rejecting the null hypothesis. Psychonomic Bulletin \& Review, 16, 225-237.

Rouder, J. N., Morey, R. D., Speckman, P. L., \& Province, J. M. (2012). Default Bayes factors for ANOVA designs. Journal of Mathematical Psychology, 56, 356-374.

Saito, S., Logie, R. H., Morita, A., \& Law, A. (2008). Visual and phonological similarity effects in verbal immediate serial recall: A test with kanji materials. Journal of Memory and Language, 59, 1-17.

Shand, M. A., \& Klima, E. S. (1981). Nonauditory suffix effects in congenitally deaf signers of American Sign Language. Journal of Experimental Psychology: Human Learning and Memory, 7, 464474.

Simpson, I. C., Mousikou, P., Montoya, J. M., \& Defior, S. (2013). A letter visual-similarity matrix for Latin-based alphabets. Behavior Research Methods, 45, 431-439.

Smyth, M. M., Hay, D. C., Hitch, G. J., \& Horton, N. J. (2005). Serial position memory in the visualspatial domain: Reconstructing sequences of unfamiliar faces. Quarterly Journal of Experimental Psychology, 58A, 909-930.

Wickelgren, W. A. (1965). Short-term memory for phonemically similar lists. American Journal of Psychology, 78, 567-574.

Publisher's note Springer Nature remains neutral with regard to jurisdictional claims in published maps and institutional affiliations. 